\title{
BMJ Open Symptoms of post-traumatic stress disorder and depression among Eritrean refugees in Ethiopia: identifying direct, meditating and moderating predictors from path analysis
}

\author{
Berhanie Getnet, ${ }^{1,2}$ Girmay Medhin, ${ }^{3}$ Atalay Alem ${ }^{1}$
}

To cite: Getnet B, Medhin G, Alem A. Symptoms of posttraumatic stress disorder and depression among Eritrean refugees in Ethiopia: identifying direct, meditating and moderating predictors from path analysis. BMJ Open 2019;9:e021142. doi:10.1136/ bmjopen-2017-021142

- Prepublication history for this paper is available online. To view these files, please visit the journal online (http://dx.doi org/10.1136/bmjopen-2017021142).

Received 25 December 2017 Revised 20 November 2018 Accepted 29 November 2018

Check for updates

(c) Author(s) (or their employer(s)) 2019. Re-use permitted under CC BY-NC. No commercial re-use. See rights and permissions. Published by BMJ.

${ }^{1}$ Department of Psychiatry, College of Health Sciences, Addis Ababa University, Addis Ababa, Ethiopia

${ }^{2}$ Department of Psychology, University of Gondar, Gondar, Ethiopia

${ }^{3}$ Aklilu Lemma Institute of Pathobiology, Addis Ababa University, Addis Ababa, Ethiopia

Correspondence to

Berhanie Getnet;

berhanie.getnet.bg@gmail.com

\section{ABSTRACT}

Objective This study aimed at testing the significance of mediating and moderating roles of sense of coherence, adaptive coping styles and social support in the relationship between exposure to trauma and psychological symptoms in a refugee population in subSaharan Africa.

Methods A cross-sectional survey design was employed to collect data. The study was carried out in Mai Aini refugee camp in Ethiopia. A total of 562 adult Eritrean refugees aged 18-74 years were selected randomly to screen for depression and post-traumatic stress disorder (PTSD) symptoms and to examine associated factors. Data were collected using the premigration and postmigration living difficulties checklist, Center for Epidemiologic Studies Depression (CES-D) scale, Primary Care PTSD Screener, coping style scale, Sense of Coherence scale and Oslo Social Support scale. Path modelling was used to test the mediation and moderation effects of prespecified factors.

Results Premigration living difficulties were associated directly with symptoms of PTSD $(\beta=0.09, p<0.05)$, and associated indirectly with PTSD symptoms in paths through duration of stay in the camp, sense of coherence, postmigration living difficulties, task-oriented coping style and depressive symptoms $(\beta=0.26, p<0.01)$. Premigration and postmigration living difficulties were associated directly with depressive symptoms with standardised estimate of $\beta=0.35(p<0.001)$ and $\beta=0.23(p<0.05)$, respectively. Postmigration living difficulties were associated indirectly with PTSD through paths of sense of coherence, task-oriented coping style and depressive symptoms $(\beta=0.13 ; p<0.01)$. Social support moderated the effect of postmigration living difficulties on depressive symptoms $(p<0.05)$. Emotion-oriented coping style moderated the effect of premigration threat for abuse on PTSD $(\beta=-0.18, p<0.001)$ and depressive $(\beta=-0.12$, $p<0.01)$ symptoms, as well as moderating threat to life on PTSD symptoms $(\beta=-0.13, p<0.001)$.

Conclusions Sense of coherence and task-oriented coping style showed a partial mediating effect on the association between exposure to trauma and symptoms of PTSD. An emotion-oriented coping style and social support moderated the effect of premigration and postmigration living difficulties, respectively. Fostering social support,
Strengths and limitations of this study

- The use of path modelling to examine multiple moderating and mediating factors on the effect of exposure to outcome variables is strength of this study.

- Rigorous adaptation of tools to the local context increased validity of the findings.

- The inherent nature of cross-sectional design employed in the current study means that a cause-effect relationship cannot be inferred.

- It is possible that study participants had reservations about reporting violations of rights and other adverse experiences in Ethiopia for fear of negative consequences.

task-oriented and emotion-oriented coping styles may be beneficial for these refugees.

\section{BACKGROUND}

In contemporary mental health research in humanitarian settings, it is important to consider the effects of traumatic events on the mental well-being of survivors. There is also increasing interest to investigate mediators of the effects of trauma on mental health, including the role of precipitating risk factors such as exposure to postmigration trauma, ${ }^{1}$ maladaptive coping methods ${ }^{2}$ and protective factors, such as a sense of coherence ${ }^{3} 4$ and adaptive coping strategies. ${ }^{5}$

Antonovsky (p19) defined sense of coherence as: 'a global orientation that expresses the extent to which one has a pervasive, enduring though dynamic feelings of confidence that (1) the stimuli deriving from one's internal and external environments in the course of living are structured and predictable and explicable;(2) the resources are available to meet the demands posed by the stimuli; and (3) these demands are challenges worthy of 
investment and engagement'. ${ }^{6}$ Sense of coherence ${ }^{7}$ and emotion-oriented coping strategies ${ }^{8}$ have been reported to moderate the effect of traumatic events. Social support is reported to be inversely associated with depression. ${ }^{9}$ Similarly, social support has also been reported to have a moderating effect when there has been exposure to trauma on the symptoms of post-traumatic stress disorder (PTSD) among Eritrean and Sudanese asylum seekers in Israel. $^{10}$

In a study of Tibetan refugees in India, subsequent development of depressive or PTSD symptoms was mediated by use of coping strategies. ${ }^{5}$ Coping in this regard refers to the psychological process with which individuals respond to stressors and life hassles which acts as a mediator between stress and psychological well-being. ${ }^{11}$ In another study where the use of different coping strategies was compared between people with PTSD and a control group, it was found that those with PTSD were more likely to use an emotion-oriented coping style in dealing with stressful events compared with those without PTSD. ${ }^{12}$ The kind of coping style could moderate (strengthen or weaken) the association between other resilience variables and PTSD symptoms. ${ }^{13}$ In the same study, an engagement coping style was hypothesised to strengthen the above associations, while a disengagement coping style weakened them. ${ }^{13} \mathrm{~A}$ similar study from the Democratic Republic of Congo indicated that the interaction between emotion-oriented coping strategies and problem-oriented coping strategies (coping flexibility) was associated with lower psychological distress. ${ }^{8}$

In population-based studies conducted in Eritrea and Iraq, sense of coherence was found to be negatively associated with PTSD symptoms ${ }^{14}$ and exposure to trauma. ${ }^{15}$ In cross-cultural studies aimed at determining the relationship between sense of coherence and coping strategies in Chinese and US study participants, sense of coherence was found to be a predictor of problem solving and avoidance coping strategies. ${ }^{16}$ In another study from Pakistan and Germany, sense of coherence played a mediating role between social support and stress. ${ }^{3}$ In a study of Palestinian emergency health workers, sense of coherence was shown to be a partial mediator on the impact of trauma for both anxiety and social dysfunction. ${ }^{4}$

In a structural equation Modelling study conducted among Zimbabwean refugees in South Africa, postmigration living difficulties mediated the association between premigration living difficulties and PTSD. ${ }^{1}$ Similarly, in a recent study that focused on the importance of postmigration stressors in an ecological model of refugees' mental health, postmigration stressors were found to consistently predict levels of distress as powerfully as exposure to premigration trauma such as exposure to violence. ${ }^{17}$ In a study in Ethiopia among internally displaced persons, some subscales of coping interacted with displacement-related traumatic events leading to a significant association with mental distress. ${ }^{18}$

Continuous migration of Eritrean youth to refugee camps of Ethiopia is occurring in large numbers for a variety of reasons including: limited employment opportunities and economic need, desire for family reunification, social pressure and perceived threat of conscription. ${ }^{19}$ As of 2013, there were 53035 refugees in four camps (namely Mai Aini, Shimelba, Adi-Harush and Hitsats).$^{20}$ Of these, $37562(70.8 \%)$ were males. Mai Aini, where this study was conducted, is the second largest camp, where 17825 refugees resided at that time. Of these, 11892 (66.7\%) were males. ${ }^{20}$ The majority were students, and most of them were unemployed before coming to Ethiopia. ${ }^{20} \mathrm{~A}$ recent update by the United Nations High Commissioner for Refugees (UNHCR) indicated that about 11718 Eritrean refugees were living in Mai Aini camp, of which 4826 $(41 \%)$ were female. ${ }^{21}$

Although there are reports on mediating and moderating roles of sense of coherence, coping styles and social support in the relationship between exposure to trauma and psychological symptoms in humanitarian settings, to our knowledge there are no such studies in Eritrean refugees living in Ethiopia. Specifically, we wanted to investigate whether: (a) a sense of coherence and task-oriented coping together mediated the relationship between exposure to premigration and postmigration living difficulties and psychological symptoms (PTSD and depression), (b) social support moderated the effect of exposure to traumatic events on symptoms of PTSD and depression, (c) the interaction between emotion-orientated and task-oriented coping styles would be associated with reduced symptoms of PTSD and depression.

\section{MATERIALS AND METHODS Study setting}

As stated earlier, this study was conducted at Mai-Aini refugee camp, $1116 \mathrm{~km}$ North of Addis Ababa, the capital city of Ethiopia. Mai-Ani is one of the refugee camps hosting Eritreans which was established in 2008 through the support given to the Ethiopian Government by the UNHCR. $^{19}$

\section{Study design}

Data for this report were collected using a cross-sectional study design.

\section{Sample size estimation}

Sample size determination was carried out considering the average prevalence of PTSD to be $30.7 \%$ among refugees in East African camps ${ }^{22-24}$ with $4 \%$ precision and a $95 \%$ CI. Adding $10 \%$ for the likely non-response, and using a single population proportion formula, the required sample size was 562 .

\section{Sampling}

According to a population census document from the Administration of Refugees and Returnees Affairs (ARRA) and UNCHR, there were a total of 10006 registered refugees living in the camp in December 2015, of whom 5749 were males. However, there was incomplete information 
about refugees' addresses, especially for the newly arrived. Thus, we allocated a house number to each household and conducted a household census from December 2015 to January 2016. The census showed that a total of 2055 households were registered in the camp, out of which 100 households were excluded because household members were minors (children below the age of 18). The remaining 1955 households were taken as a sampling frame. From this sampling frame, 562 households were selected using the simple random sampling method and one participant was interviewed by a trained data collector from each household. In a household where there were more than one eligible persons (18 years old and above and having Eritrean nationality before migrating to Ethiopia), one participant was selected using the lottery method. Twenty-two of the selected households were replaced because household members were not found after three visits by data collectors. The replacement was done using neighbouring households (ie, from those that preceded or followed the selected households' numbers). Data collection took place from January to March 2016. As the principal investigator (BG) was supervising and monitoring the data collection on daily basis, incomplete data were collected by sending the data collectors back to the respondents' homes.

\section{Variables}

Symptoms of PTSD and depression were the main outcome variables in this study. Premigration and postmigration living difficulties were the exposures and main predictor variables. Sense of coherence, task-oriented coping, emotion-oriented, avoidance-oriented coping styles and social support were assumed to moderate the effect of exposure to traumatic events on symptoms of depression and PTSD. Variables such as gender, age and marital status, level of education, frequency of prayer and duration of stay in the refugee camp were regarded as potential confounders.

\section{Instrument adaptation}

All instruments used in this study were adapted following standard adaptation procedures for transcultural study. ${ }^{25}$ Except for the Center for Epidemiologic Studies Depression (CES-D) scale, all instruments were translated from the source language (English) into the target language (Tigrigna) by two bilingual experts, followed by masked back translated into English by two other independent bilingual translators. The translations and the back translations were given to an expert panel whose mother tongue was Tigrigna, the language used for the study. Taking comments from the experts as an input, consensus meetings were held to merge the two translated versions by the translators. The translations were then rated using a 4-point rating scale for their content relevance by seven experts to obtain a Content Validity Index. ${ }^{26}{ }^{27}$ Following that, cognitive interviews were conducted with six refugees not included in the study to ensure the understandability, as well acceptability, of items for the target Eritrean refugee community. Minor revisions were made based on their feedback. All instruments were pilot tested before they were employed in the main study.

\section{Patient and public involvement}

Since this study involved members of vulnerable population, who were at risk of violation of their rights and susceptible to various health problems including mental health problems, the necessary precautions were taken when recruiting participants for the study. The research question and adaptation of outcome measures were informed by literature review. The preferences and experiences of refugees were understood based on a situational analysis study (unpublished data) carried out in the same community 1 year prior to the current study (B Getnet, personal communication, 2015). The situational analysis study involved interviews with key informants among the refugees and focus group discussion with refugee counsellors and Eritrean lay counsellors trained in psychosocial interventions. The participants were assured about legal protection for their security. Permission letters were also obtained from concerned higher authorities, ARRA in Addis Ababa and its site offices, before participants gave us their informed consent. High scorers on PTSD and depression symptom scales were encouraged to visit refugee counsellors or to obtain psychiatric care from local healthcare staff. Findings of this study will be disseminated to the research participants through workshops and briefing sessions.

\section{Measures}

Traumatic events were measured using the Pre and Post Migration Living Difficulties Checklist. ${ }^{1}$ This tool was developed and used to measure traumatic events of Zimbabwean refugees in South Africa in premigration and postmigration periods. It has a 14 -item checklist with five point response format (ie, strongly disagree score $=1$, disagree $=2$, neutral $=3$, agree $=4$ and strongly agree $=5$ ). Examples of items include: 'I was beaten and harassed; I was sexually harassed'. In order to differentiate those who had encountered trauma from those who had not, the authors re-coded responses 1 to 3 as 0 and 4 to 5 as $1 .^{28}$ In the pilot study $(n=50)$, internal consistency alpha values of 0.86 and 0.83 were obtained for premigration and postmigration living difficulties, respectively. Confirmatory factor analysis (CFA) in the main study sample $(n=562)$ indicated that the two factors structure of premigration living difficulties had an acceptable fit to the present data: $\chi^{2}=172.444 ; \chi^{2} / \mathrm{df}=2.874$; Comparative Fit Index $(\mathrm{CFI})=0.971$; Tucker Lewis Index (TLI) $=0.951$; Root Mean Square Error of Approximation (RMSEA) $=0.058$ $(90 \% \mathrm{CI}=0.048$ to 0.068$)$; Standardized Root Mean Residual $($ SRMR $)=0.0402$. In this model, the first factor, threat for life (9-items), and the second factor, threat for abuse (5-items), had good internal consistency (Cronbach's alpha 0.88 and 0.82 , respectively). Two factor structures of postmigration living difficulties have demonstrated acceptable fit to the present data: $\chi^{2}=202.576 ; \chi^{2} /$ 
$\mathrm{df}=3.554 ; \mathrm{CFI}=0.950 ; \mathrm{TLI}=0.919 ; \mathrm{RMSEA}=0.067(90 \% \mathrm{CI}$ 0.058 to 0.078$)$. The first factor, threat for life (9-items) and the second factor, threat for abuse (5-items) are internally consistent (Cronbach's alpha values 0.68 and 0.72 , respectively). Besides, the Item-level Content Validity Index (I-CVI) for the 14 items in the present study ranged from 0.86 to 1.00 . The Scale-level Average Content Validity Index (S-CVI/Ave) was found to be 0.98 .

Depression was measured using the CES-D. ${ }^{29}$ The English version of CES-D is a 20 item scale with four alternative response options: none of the time, a little of the time, a moderate amount of the time and most of the time, to be scored 0 to $3 .^{29}$ One example of an item reads: 'I was bothered by things that usually do not bother me'. This instrument is designed to measure depressive symptomatology in the general population. ${ }^{29}$ The CES-D was translated into Tigrigna and validated for Tigrigna speaking Eritrean refugees in the USA, and the adaptation study found an alpha value of 0.86 for internal consistency and 0.91 for test re-test reliability. ${ }^{30}$ The internal consistency in our pilot study was $0.92(n=50)$ and 0.91 in the main study $(n=562)$. After allowing unique values to correlate, CFA suggested that the present data best fitted with two dimensions of negative affect and positive affect with a second order single factor model $\left(\chi^{2}=271.65 ; \mathrm{df}=144 ; \mathrm{CFI}=0.975\right.$; TLI=0.967; SRMR=0.0378; RMSEA=0.04). All the 20 items loaded sufficiently onto either of these two dimensions better than other computing factor structures of CES-D in the literature. Thus, composite reliability alpha of the two factors negative affect (16 Items) had an alpha value of 0.93, whereas the positive affect (four items) had an alpha value of $0.70(n=562)$. The I-CVI values for the 20 items ranged from 0.71 to 1.00 and S-CVI/Ave for the total scale was found to be 0.92 .

PTSD was measured using the Primary Care PTSD Screener (PC-PTSD). ${ }^{31}$ This is a four item brief screening instrument, having two response options, 'Yes' or 'No'.31 This scale has one common instruction for all items, 'In your life, have you ever had any experience that was so frightening, horrible or upsetting that, in the past month...'. One example item is: 'Have had nightmares about it or thought about it when you did not want to?'31 Test re-test reliability was found to be 0.83 . $^{32}$ Sensitivity and specificity of PC-PTSD was 0.78 and 0.87 , respectively. $^{31}$ The scale has been extensively used to study PTSD in veterans in United States, ${ }^{31} 32$ Afghanistan and $\mathrm{Iraq}^{33}$; its use for screening PTSD in refugees is also documented. ${ }^{3435}$ In the present study, the internal consistency alpha value was found to be 0.68 in the pilot study $(n=50)$ and 0.64 in the main study $(n=562)$. Item-total correlation for the four items ranged from 0.59 to 0.75 in the pilot study. For the four items, I-CVI was 1 which makes the overall Scale level Item Validity Index to be 1 . Principal component analysis showed that the four items loaded on a single factor (ranging from 0.58 to 0.82 ). Similarly, CFA showed that all the four items, each of which basically represents one factor structure of PTSD, loaded adequately onto a single dimension, ranging from 0.43 (numbing) to 0.78 (re-experiencing). The present data showed closer fit with a single factor structure of PTSD with fit indices of $\chi^{2}=17.275 ; \chi^{2} / \mathrm{df}=8.622$, $\mathrm{CFI}=0.963$; TLI $=0.888$; $\mathrm{RMSEA}=0.117(90 \%$ CI 0.070 to $0.17), \mathrm{SRMR}=0.0355$.

Coping strategies were measured using a Coping Style Scale $^{18}$ which consists of 10 items. The items require participants to respond 'this is not like me' or 'this is like me'. ${ }^{18}$ The first set of 4-items follow one scenario: 'Imagine, someone is spreading damaging gossip about you'. One example of the item that follows this reads: 'Some people would try to find the person who began the gossip and discuss it with him/her'. ${ }^{18}$ The second set of 6-items follow an imagined scenario 'Imagine that some property of yours, money, food or object of value is lost or stolen', one example of items following this is 'some people would avoid thinking about the loss or theft'. ${ }^{18}$ This instrument was cross-culturally validated by Trans-cultural Psychosocial Organization, and later used to study coping strategies from among displaced Ethiopians from Eritrea during the secession of Eritrea from Ethiopia. $^{18} 36$ This scale roughly captured three coping strategies which include task-oriented, avoidance-oriented and emotion-oriented coping strategies. ${ }^{18}$ In the present study, the internal consistency alpha value was found to be 0.61 in the pilot study $(n=50)$ and 0.48 in the main study $(n=562)$. The three factors of coping have demonstrated reasonable fit to the present data with exclusion of item $8(\beta=0.014, p>0.05)$. The remaining nine items had a close fit to the data: $\chi^{2}=54.549 ; \chi^{2} / \mathrm{df}=2.273$; CFI $=0.901$; TLI $=0.851$; RMSEA $=0.048$ (90\% CI 0.031 to 0.064 ).

Resilience was measured using the Sense of Coherence Scale (SoC-13). ${ }^{37}$ This is a 13-item semantic differential scale adapted for Eritrean culture in the form of a 5-point Likert scale from the original 7-point scale to reduce complexity. ${ }^{14}$ For example, item 1 asks, 'Do you have a feeling that you don't really care about what goes on around you?' Response options range from 'very seldom or never' to be scored as 1 and 'very often' to be scored as $5 .{ }^{14}$ Responses for the items 1, 2, 3, 7 and 10 were reverse coded as per previous study. ${ }^{38}$ The instrument has been reported to be an adequate measure of resilience in an Eritrean population. ${ }^{14}$ Despite the conflicting findings regarding the dimensionality of SoC-13, ${ }^{39}$ our data in the present study fitted well into a single factor structure, except for item 2. Twelve items demonstrated significant loadings onto a single factor $\left(\chi^{2}=57.54 ; \mathrm{df}=34 ; \chi^{2} / \mathrm{df}=1.69\right.$; $\mathrm{CFI}=0.98$; RMSEA=0.035 (90\% CI=0.02 to 0.05$)$ which gave supporting evidence that it measured a one-dimensional construct as proposed by the original scale developer. ${ }^{37}$ Cronbach's alpha value for internal consistency in the pilot study $(n=52)$ and the main study $(n=562)$ were 0.67 and 0.74 , respectively.

Social support was measured using the Oslo Social Support Scale (OSS-3). ${ }^{9}$ This is a scale consisting of three items in which the sum of scores ranges from 3 to $14 .^{40}$ In a validation study of OSS-3 in Nigeria, Cronbach's alpha value for internal consistency was found to be 
$0.5 .^{40}$ In our pilot study ( $\left.\mathrm{n}=52\right)$, Cronbach's alpha value was 0.39 and the alpha value for the main study was 0.58 $(\mathrm{n}=562)$. CFA indicated that all the items significantly and adequately loaded onto a single factor ranging from 0.49 to 0.60 , with fit indices of $\chi^{2}=4.233, \chi^{2} / \mathrm{df}=4.233$, $\mathrm{CFI}=0.978, \mathrm{TLI}=0.934, \mathrm{RMSEA}=0.076 \quad(90 \% \mathrm{CI}=0.014$ to 0.156). All the items received I-CVI of 1 which makes S-CVI to be 1 for the total scale.

\section{Statistical analysis}

Associations and direction of associations tested in this study were based on the existing literature. Path analysis was performed to test the significance of association and to identify direct and indirect effects of variables using IBM SPSS Amos V.21. The effect of interaction among some coping subscales with subscales of premigration and postmigration living difficulties was calculated by multiplying the respective variables; thereby interaction terms were created. Then, the effect of each interaction term was tested for its significance of association with PTSD or depression symptoms using independent path analysis. For Item Level Content Index (I-CVI), values of 0.78 or higher are considered to be valid indices for content validity if the instrument is rated by $6-10$ experts. ${ }^{26}$ In the present study, the cut-off values for fit indices of an acceptable model included values of $\chi^{2} / \mathrm{df}$ less than 3 , greater than or equal to 0.95 for CFI, greater than or equal to 0.95 for TLI, less than or equal to 0.06 to 0.08 for RMSEA and less than or equal to 0.08 for SRMR. ${ }^{41}$

\section{RESULTS}

\section{Sociodemographic characteristics of participants}

Of the 562 participants (table 1), 304 (54.1\%) were females. Age ranged from 18 to 74 years (with mean age $=29.6, \mathrm{SD}=10.18$ ), the majority was literate and the average duration of stay in the refugee camp was 3.71 years. A high proportion of participants belonged to the Tigrinya ethnic group (92\%). Very few participants came from Saho, Bilen, Tigre and Jabelty ethnic groups of Eritrea (constituting 8\% altogether). Eighty-five per cent were followers of Coptic Orthodox Christianity. Participants had a diverse profile of occupations before coming to Ethiopia, but students, ex-soldiers and farmers constituted $71 \%$.

\section{Direct and mediating predictors of PTSD and depression symptoms}

The path model as shown in figure 1 fitted well with the data: $\quad\left(\chi^{2}=39.97 ; \mathrm{df}=24 ; \chi^{2} / \mathrm{df}=1.67 ; \mathrm{CFI}=0.985\right.$; TLI $=0.972 ;$ SRMR $=0.05$; RMSEA $=0.034(90 \%$ CI 0.013 to 0.053$) ; \mathrm{p}=0.022$ ). In examining the paths in detail, both premigration $(\beta=-0.19, \mathrm{p}<0.001)$ and postmigration $(\beta=-0.15, p<0.001)$ traumatic events were significantly associated with sense of coherence. Sense of coherence in turn was significantly and negatively associated with symptoms of PTSD $(\beta=-0.16, p<0.001)$ and depression $(\beta=-0.40, p<0.001)$ (table 2$)$. Premigration

\begin{tabular}{|c|c|c|}
\hline \multicolumn{2}{|c|}{ Characteristics } & \multirow{2}{*}{$\frac{\mathbf{N}(\%)}{258(45.9)}$} \\
\hline Sex & Male & \\
\hline & Female & $304(54.1)$ \\
\hline \multirow[t]{7}{*}{ Age } & Mean (SD) & $29.6(10.2)$ \\
\hline & $18-24$ & $205(36.5)$ \\
\hline & $25-34$ & $219(39.0)$ \\
\hline & $35-44$ & $89(15.8)$ \\
\hline & $45-54$ & $29(5.2)$ \\
\hline & $55-64$ & $15(2.5)$ \\
\hline & $65-74$ & $5(0.9)$ \\
\hline \multirow{4}{*}{$\begin{array}{l}\text { Educational } \\
\text { background }\end{array}$} & Non-literate & $67(11.9)$ \\
\hline & Elementary school & 232 (41.3) \\
\hline & Secondary school & $238(42.3)$ \\
\hline & College graduate or above & $25(4.5)$ \\
\hline \multirow[t]{4}{*}{ Marital status } & Single & 189 (33.6) \\
\hline & Married & $327(58.2)$ \\
\hline & Divorced & $29(5.2)$ \\
\hline & Widowed & $17(3.0)$ \\
\hline \multirow[t]{5}{*}{ Religion } & Orthodox & 477 (84.9) \\
\hline & Protestant & $17(3.0)$ \\
\hline & Catholic & $23(4.1)$ \\
\hline & Muslim & $44(7.8)$ \\
\hline & Jehovah Witness & $1(0.2)$ \\
\hline \multirow{7}{*}{$\begin{array}{l}\text { Past } \\
\text { occupation in } \\
\text { Eritrea }\end{array}$} & Student & $201(35.8)$ \\
\hline & Military & $111(19.8)$ \\
\hline & Farmer & $89(15.8)$ \\
\hline & Home maid & $66(11.7)$ \\
\hline & Educator & $23(4.1)$ \\
\hline & Daily labourers & $15(2.7)$ \\
\hline & Others & 57 (10.1) \\
\hline
\end{tabular}

living difficulties were directly associated with symptoms of PTSD $(\beta=0.09, p<0.05)$ and with depression $(\beta=0.35, p<0.001)$. It was also indirectly associated with depression via sense of coherence and postmigration living difficulties. Postmigration living difficulties were directly associated with depression symptoms $(\beta=0.23, \quad p<0.001)$ and indirectly associated with PTSD symptoms via sense of coherence, symptoms of depression and task-oriented coping style.

Duration of stay in the refugee camp, sense of coherence, postmigration living difficulties, task-oriented coping style and depression together mediated the association between premigration living difficulties and PTSD symptoms (standardised indirect coefficient $=0.26$, $\mathrm{p}<0.01$ ). Similarly, task-oriented coping style, sense of coherence and depression symptoms together mediated the association between postmigration living difficulties 


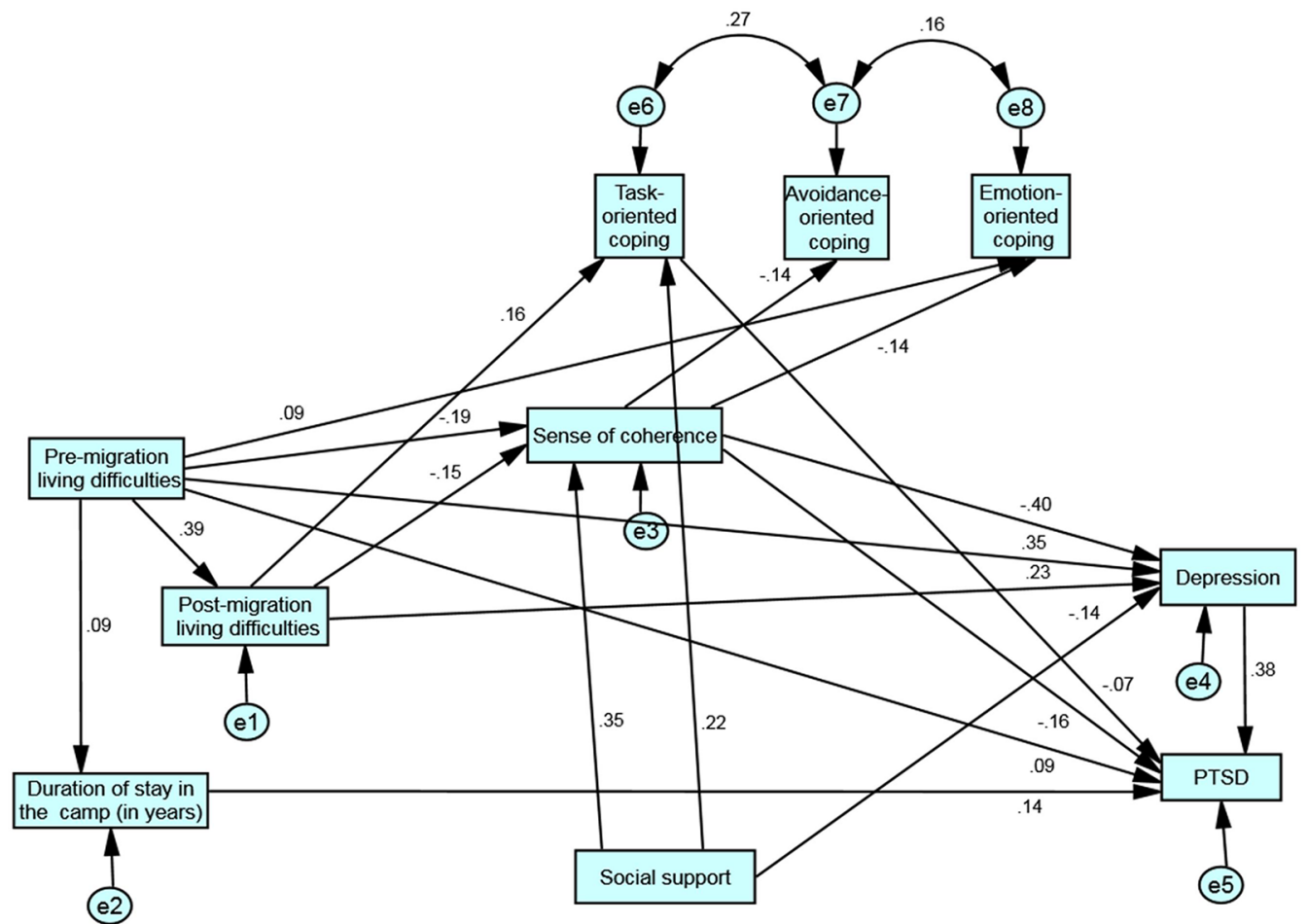

Figure 1 Path model depicting direct and indirect (mediated) association between exposure to trauma and mental health outcomes. All paths are depicting standardised significant coefficients. Rectangles represent observed variables, circles represent disturbance (error terms), one-sided arrows together with coefficient values are equivalent to regression beta weights between the two variables connected by these arrows, double-headed arrows represent co-variances between error terms. PTSD, post-traumatic stress disorder.

and PTSD symptoms (standardised indirect coefficient $=0.13, \mathrm{p}<0.01) \quad($ table 3$)$.

Premigration and postmigration living difficulties were not significantly associated with social support. Hence, social support was not a mediator for the association between exposure to trauma and symptoms in this path model (see figure 1).

The association between premigration living difficulties and depression was strengthened by exposure to postmigration living difficulties $(\beta=0.23, p<0.001)$. Premigration living difficulties were significantly and positively associated with emotion-oriented coping style $(\beta=0.09, p<0.05)$ as well as postmigration living difficulties $(\beta=0.39, p<0.001)$. On the other hand, postmigration living difficulties were associated positively with task-oriented coping style $(\beta=0.16$, $\mathrm{p}<0.001)$. Depression partially mediated and strengthened the association between postmigration trauma and symptoms of PTSD $(\beta=0.38, \mathrm{p}<0.001)$.

\section{Moderators}

Test for moderation (interaction effect) (table 4) illustrates that sense of coherence and task-oriented coping style did not moderate the effect of premigration/postmigration living difficulties on PTSD or depression symptoms $(p>0.05)$. However, the interaction between social support and postmigration living difficulties resulted in an inverse association with depression $(\mathrm{p}<0.05)$ which shows a moderating role of social support on the effect of postmigration living difficulties on depression symptoms.

Emotion-oriented coping style appeared to have a moderating effect on PTSD symptoms, with an interaction with the threat for life subscale of premigration living difficulties both in females $(\beta=-0.120, p<0.05)(n=304)$ and males $(\beta=-0.114, p<0.05)(n=258)$ (table 5). Similarly, the interaction between emotion-oriented coping styles with the threat for abuse subscale of premigration trauma was negatively and significantly associated both with PTSD and depression symptoms (model-6). Subgroup analysis by gender (table 5: models 7-12) showed that exposure to postmigration trauma was associated with symptoms of PTSD only in females $(\mathrm{p}<0.001)$.

Task-oriented coping style was independently and negatively associated with PTSD (table 5: model-1), while emotion-oriented coping style showed a positive and independent association with depression (table 5: models 9, 12 and 13). The interaction between emotion-oriented and task-oriented coping styles was negatively and significantly associated with depression both in females $(\beta=-0.12$, $\mathrm{p}<0.05)$ and males $(\mathrm{B}=-0.15, \mathrm{p}<0.05)$. Emotion-oriented coping had an inverse association with symptoms when it interacted with other variables (table 5: model 3, 6 and 13). An avoidance-oriented coping style was positively associated with symptoms of depression for females when tested as an independent predictor (table 5: models 8 and 11). 
Table 2 Path coefficients between predictors and endogenous variables in the path model

\begin{tabular}{|c|c|c|c|c|c|}
\hline Predictors & Endogenous variables & $\begin{array}{l}\text { Unstandardised } \\
\text { estimate }(95 \% \mathrm{Cl})\end{array}$ & SE & $\begin{array}{l}\text { Standardised } \\
\text { path coefficient }\end{array}$ & $P$ value \\
\hline Premigration difficulties & Postmigration & 0.21 (0.17 to 0.25$)$ & 0.021 & 0.39 & $<0.001$ \\
\hline Postmigration difficulties & Sense of coherence & $-0.21(-0.32$ to -0.10$)$ & 0.056 & -0.15 & $<0.001$ \\
\hline Premigration difficulties & Sense of coherence & $-0.14(-0.20$ to -0.08$)$ & 0.031 & -0.19 & $<0.001$ \\
\hline Social support & Sense of coherence & 1.28 (1.01 to 1.55$)$ & 0.139 & 0.35 & $<0.001$ \\
\hline Sense of coherence & Depression & $-0.59(-0.68$ to -0.50$)$ & 0.046 & -0.40 & $<0.001$ \\
\hline Premigration difficulties & Depression & 0.38 (0.31 to 0.45$)$ & 0.034 & 0.35 & $<0.001$ \\
\hline Postmigration difficulties & Depression & 0.46 (0.34 to 0.58$)$ & 0.062 & 0.23 & $<0.001$ \\
\hline Premigration difficulties & Duration of stay & 0.02 (0.00 to 0.04$)$ & 0.011 & 0.09 & $<0.05$ \\
\hline Social support & Depression & $-0.78(-1.05$ to -0.51$)$ & 0.162 & -0.14 & $<0.001$ \\
\hline Postmigration difficulties & Task-oriented coping & 0.03 (0.01 to 0.05$)$ & 0.008 & 0.16 & $<0.001$ \\
\hline Social support & Task-oriented coping & $0.13(0.09$ to 0.17$)$ & 0.022 & 0.22 & $<0.001$ \\
\hline Duration of stay & PTSD & 0.07 (0.04 to 0.10$)$ & 0.017 & 0.14 & $<0.001$ \\
\hline Sense of coherence & Avoidance-oriented coping & $-0.01(-0.02$ to -0.002$)$ & 0.004 & -0.14 & $<0.001$ \\
\hline Sense of coherence & PTSD & $-0.03(-0.04$ to -0.02$)$ & 0.007 & -0.16 & $<0.001$ \\
\hline Premigration difficulties & PTSD & 0.01 (0.00 to 0.02 ) & 0.005 & 0.09 & $<0.05$ \\
\hline Depression & PTSD & 0.04 (0.03 to 0.05$)$ & 0.006 & 0.38 & $<0.05$ \\
\hline Task-oriented coping & PTSD & $-0.07(-0.14$ to -0.004$)$ & 0.038 & -0.07 & $<0.05$ \\
\hline Premigration difficulties & Emotion-oriented coping & 0.01 (0.004 to 0.02) & 0.003 & 0.09 & $<0.001$ \\
\hline Sense of coherence & Emotion-oriented coping & $-0.01(-0.02$ to -0.002$)$ & 0.004 & -0.14 & $<0.01$ \\
\hline
\end{tabular}

PTSD, post-traumatic stress disorder.

\section{DISCUSSION}

In the present study that aimed to investigate the mediating and moderating roles of theoretically relevant factors (ie, sense of coherence, adaptive coping style and social support) between exposure to traumatic events and psychological symptoms, findings supported our alternative hypotheses. Sense of coherence partially mediated (but did not moderate) the effect of exposure to premigration traumatic events and postmigration traumatic events on symptoms of PTSD and depression. The same effect was shown with a task-oriented coping style which partially mediated (but did not moderate) the effect of exposure to postmigration traumatic events on PTSD symptoms. However, social support moderated the effect of postmigration living difficulties on symptoms of depression, whereas use of emotion-oriented coping strategies moderated the effect of exposure to premigration traumatic events on symptoms of PTSD and depression.

Our finding that the duration of stay in the refugee camp exacerbated symptoms is consistent with previous findings in Australia ${ }^{42}$ where prolonged detention and temporary protection of refugees contributed substantially to the

Table 3 Mediation analysis between exposure to trauma during premigration and postmigration period and symptoms (PTSD and depression)

\begin{tabular}{|c|c|c|c|c|}
\hline Hypothesised relationship & Mediating variables & $\begin{array}{l}\text { Standardised } \\
\text { direct effect }\end{array}$ & $\begin{array}{l}\text { Standardised } \\
\text { indirect effect }\end{array}$ & Result \\
\hline $\begin{array}{l}\text { Premigration living } \\
\text { difficulties } \rightarrow \text { PTSD }\end{array}$ & $\begin{array}{l}\text { Duration of stay in the camp, sense of } \\
\text { coherence, postmigration living difficulties, } \\
\text { task-oriented coping, depression. }\end{array}$ & $0.09^{*}$ & $0.26^{\star *}$ & Partially mediated \\
\hline $\begin{array}{l}\text { Postmigration living } \\
\text { difficulties } \rightarrow \text { PTSD }\end{array}$ & $\begin{array}{l}\text { Task-oriented coping, sense of coherence } \\
\text { and depression. }\end{array}$ & -0.05 (n.s.) & $0.13^{* *}$ & Partially mediated \\
\hline $\begin{array}{l}\text { Postmigration living } \\
\text { difficulties } \rightarrow \text { depression }\end{array}$ & Sense of coherence. & $0.23^{\star \star \star}$ & $-0.06^{\star \star}$ & Partially mediated \\
\hline
\end{tabular}

${ }^{*} \mathrm{P}<0.05 ;{ }^{* *} \mathrm{P}<0.01 ;{ }^{* \star *} \mathrm{P}<0.001$.

n.s.=not significant; PTSD, post-traumatic stress disorder. 
Table 4 Moderation analysis for sense of coherence, task-oriented coping style and social support

\begin{tabular}{|c|c|c|c|}
\hline \multicolumn{2}{|r|}{ Model main and interaction predictors } & \multirow{2}{*}{$\begin{array}{l}\text { Standardised path coefficient } \\
\text { to PTSD }(n=562) \\
0.248^{* \star \star}\end{array}$} & \multirow{2}{*}{$\begin{array}{l}\begin{array}{l}\text { Standardised path coefficient } \\
\text { to depression }(\mathbf{n}=562)\end{array} \\
0.440^{\star \star \star}\end{array}$} \\
\hline 1 & Premigration living difficulties & & \\
\hline & Premigration living difficulties $x$ sense of coherence & 0.057 & 0.022 \\
\hline & Sense of coherence & $-0.372^{\star \star \star}$ & $-0.513^{\star \star \star}$ \\
\hline \multirow[t]{3}{*}{2} & Postmigration living difficulties & $0.103^{\star *}$ & $0.371^{\star \star \star}$ \\
\hline & Postmigration living difficulties $x$ sense of coherence & -0.036 & 0.053 \\
\hline & Sense of coherence & $-0.407^{\star \star \star}$ & $-0.536^{\star \star *}$ \\
\hline \multirow[t]{3}{*}{3} & Premigration living difficulties & $0.353^{\star \star \star}$ & $0.547^{\star \star \star}$ \\
\hline & Premigration living difficulties $\mathrm{x}$ task-oriented coping & 0.027 & -0.004 \\
\hline & Task-oriented coping & $-0.092^{*}$ & -0.012 \\
\hline \multirow[t]{3}{*}{4} & Postmigration living difficulties & $0.221^{* \star *}$ & $0.466^{\star \star \star}$ \\
\hline & Postmigration living difficulties $\mathrm{x}$ task-oriented coping & -0.019 & -0.061 \\
\hline & Task-oriented coping & -0.076 & 0.000 \\
\hline \multirow[t]{3}{*}{5} & Premigration living difficulties & $0.327^{\star \star \star}$ & $0.536^{\star \star \star}$ \\
\hline & Premigration living difficulties $\mathrm{x}$ social support & 0.037 & -0.006 \\
\hline & Social support & $-0.249^{\star \star \star}$ & $-0.295^{\star \star \star}$ \\
\hline \multirow[t]{3}{*}{6} & Postmigration living difficulties & $0.193^{\star \star \star}$ & $0.445^{\star * \star}$ \\
\hline & Postmigration living difficulties $x$ social support & -0.029 & $-0.106^{\star}$ \\
\hline & Social support & $-0.253^{\star \star \star}$ & $-0.259^{\star \star \star}$ \\
\hline
\end{tabular}

${ }^{*} \mathrm{P}<0.05 ;{ }^{* \star} \mathrm{P}<0.01 ;{ }^{\star \star \star} \mathrm{P}<0.001$.

PTSD, post-traumatic stress disorder.

risk of PTSD, depression and other mental health-related disabilities. The likely explanation for the exacerbating effect of longer duration of stay for worsening symptoms of PTSD symptoms in the present study is that the longer refugees stay in the camp, the greater the chance to be exposed to different postmigration traumas and abuses. Meagre support, limited employment opportunities and resources and being in this situation for a prolonged period are also other stressors that could lead them to experience some form of psychological symptoms. As refugees stay longer in the camp, their chance of leaving the camp might decrease overtime, resulting in a sense of hopelessness that exacerbated their psychological symptoms.

The finding of increased use of emotion-oriented and task-oriented coping styles with increased exposure to premigration and postmigration living difficulties, respectively, is in line with previous findings of Tibetan refugees in India. ${ }^{5}$ The indirect (mediated) effect of premigration living difficulties on depression and PTSD symptoms via the path of postmigration living difficulties seen in the present study has theoretical support in the literature. ${ }^{117}$ The association between premigration living difficulties and PTSD was mediated by postmigration living difficulties among Zimbabwean refugees in South Africa. ${ }^{1}$ A recent study has also reported that postmigration stressors accounted for greater variance in the level of depression and anxiety compared with experiences of trauma and loss. ${ }^{17}$ This indicates that being exposed to premigration trauma alone is not the sole factor affecting the mental well-being of refugees, but postmigration living circumstances are also likely to play an intervening (mediating) role. The mediating role of postmigration living difficulties in the present study can be best explained by the continuous exposure of Eritrean refugees to stressful conditions of the camp life in Ethiopia, like poverty and economic dependency, poor and overcrowded housing. These experiences are likely to worsen the effect of exposure to traumatic events in their home country before migration.

The association between sense of coherence and reduced symptoms of PTSD and depression in this study is in line with reports from other studies that describe an inverse association between sense of coherence and psychological distress in many cultural contexts. ${ }^{3} 141537$ On the other hand, sense of coherence mediated the association between traumatic events and psychological symptoms in this study which is again in agreement with previous findings. ${ }^{34}$ However, its moderating role between exposure to trauma and psychological symptoms reported in other studies ${ }^{7}$ was not found in this study.

The moderating role of social support seen in this study is in line with evidence of previous studies. ${ }^{10}$ However, this is unlike the recent report which indicated that perceived social support had a moderating effect of exposure to trauma on symptoms of PTSD among Eritrean and Sudanese asylum seekers in Israel. ${ }^{10}$ The current findings showed that social support moderated the effect 
응

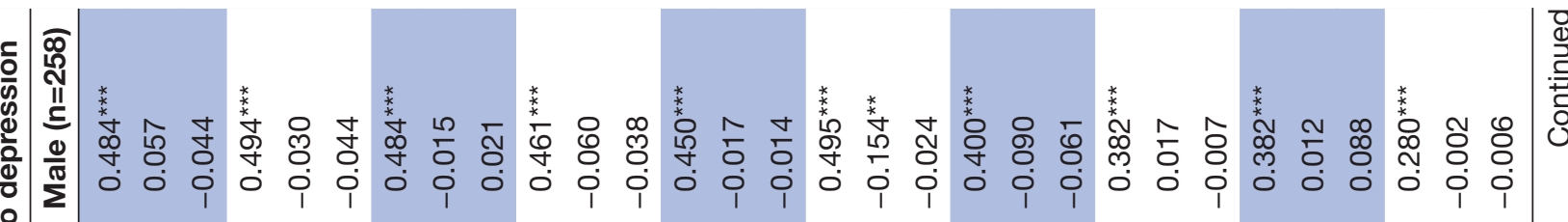

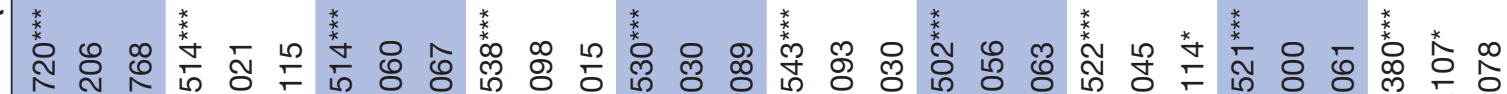

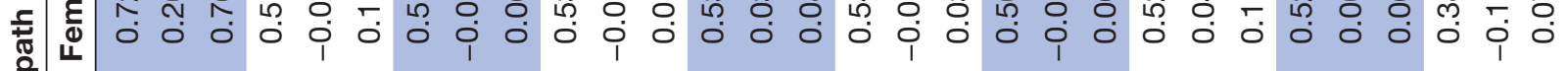

के

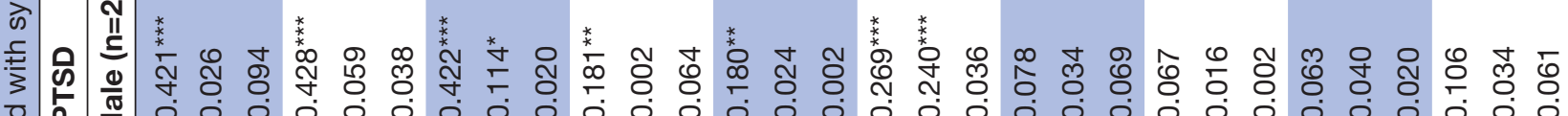

秋

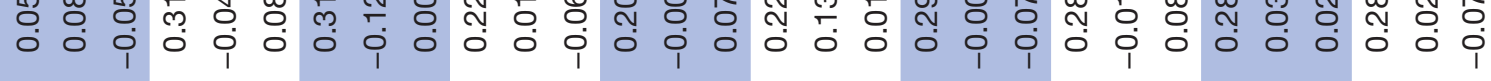

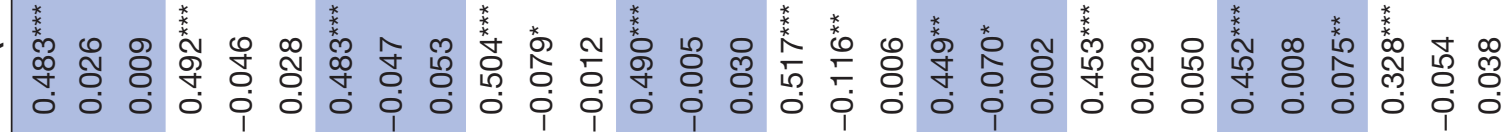

类

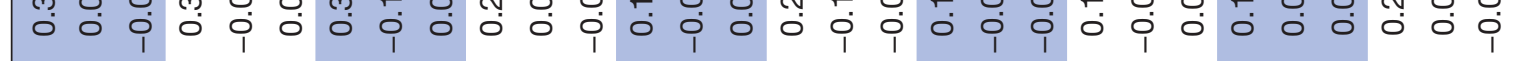

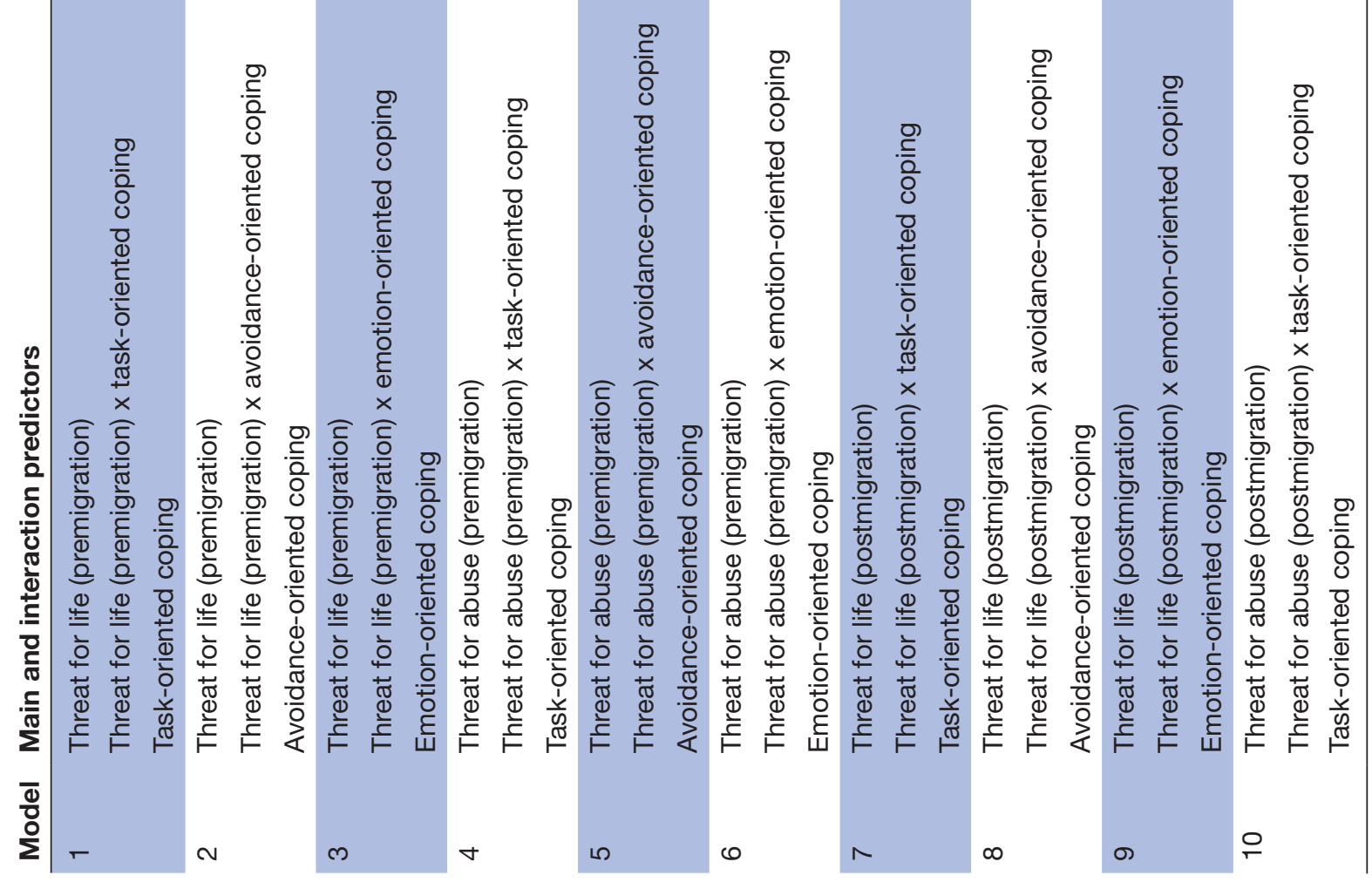


of exposure to postmigration trauma on symptoms of depression. The absence of a mediating role of social support between exposure to trauma and symptoms of PTSD and depression is in agreement with findings from a study conducted in African-Americans aged between 18 and 54 in the National Co-morbidity Study which reported that social support did not mediate the effects of financial strain or traumatic events on psychological symptoms. ${ }^{43}$

A task-oriented coping style mediated the relationship between exposure to postmigration trauma and symptoms of PTSD in this study which signifies the importance of this coping strategy for Eritrean refugees. This finding is also consistent with findings in Tibetan refugees in India. ${ }^{5}$ However, use of a task-oriented coping style did not have the moderating effect of exposure to trauma on psychological health indicated in previous studies, including the findings from displaced Ethiopians from Eritrea who were living in temporary shelters in Ethiopia. ${ }^{18}$ An avoidance-oriented coping style was not found to be associated with lower symptoms in this studies, in contrast to findings from previous studies from Ethiopian ${ }^{18}$ and Congolese sample, ${ }^{8}$ where an avoidance-coping strategy was found to be beneficial. The likely explanation for this is the composition of the refugee participants: most were literate, with an urban-orientation.

The strong association between depression and PTSD symptoms supported what was reported in the Zimbabwean sample. ${ }^{1}$ However, since our finding was based on a cross-sectional study, it is noteworthy regarding the direction of causality because of the possibility that elevated symptoms of depression might have been driven by heightened symptoms of PTSD or vice-versa. The inverse association between the interaction term (ie, emotion-oriented coping style and task-oriented coping style) and reduced symptoms of depression for both sexes seen in the present study has supported one of our hypotheses, and this finding is in line with the report from the Democratic Republic of Congo. ${ }^{8}$

Our findings that PTSD symptoms are differentially associated with postmigration trauma in females, not in males, may imply the ongoing gender-based vulnerability of female refugees to postmigration abuses in the refugee camps which are also reported in previous studies conducted in refugee camps in Ethiopia. ${ }^{44}$ Particularly, gender-based physical violence was reported in a previous study from Eritrean refugee population living in Shimelba, one of the biggest refugee camps in Northern Ethiopia. ${ }^{44}$ Another study in Somali female refugees reported that they were vulnerable to rape during transit and abduction in the refugee camp in South Eastern Ethiopia. ${ }^{45}$ The premigration/postmigration living difficulties in this study should be understood with caution because Eritrean refugees travelled only a short distance to cross the border to a neighbouring country that has a similar culture. The essence of premigration/postmigration living difficulties described in the literature denotes something different for refugees who have travelled long distances and those who have entirely 
different culture in comparison with the culture of the host country.

The association between emotion-oriented coping style and reduced symptoms of PTSD, when it interacted with the threat for life subscale of premigration difficulties may imply that this coping style has a potential benefit for those persons who encountered life threatening trauma. This coping strategy may help to release traumatic memories, thereby reducing PTSD symptoms. The moderating effect of emotion-oriented coping strategy for premigration abuse (sexual and physical) in reducing symptoms (PTSD and depression) implies its potential use for refugees who had experienced abuse in Eritrea to deal with symptoms of both conditions. The protective role of this coping style was also reported by previous studies in other African humanitarian settings. ${ }^{818}$

\section{Implications of the findings}

The differential association between PTSD symptoms and postmigration living difficulties for females, unlike males, may necessitate intervention for ongoing postmigration traumatic abuses uniquely for female refugees. This finding also informs national and international policy-makers for special protection of women refugees from violation of their human rights.

The current finding that duration of stay in camp mediated the effect of premigration trauma on PTSD calls for accelerated resettlement, repatriation or integration of Eritrean refugees within the host society. Enhancement of social support for the refugees also seems important because this study has shown that it has a fairly strong positive association with increased sense of coherence which in turn is associated with decreasing symptoms of post traumatic stress disorder (PTSD) and depression. Future studies should focus on longitudinal study designs to replicate the present findings from Eritrean samples in humanitarian settings.

Acknowledgements Our appreciation primarily goes to a senior Ethiopian mental health researcher, Dr Teshome Shibre kelkilie, who is presently living in Canada, for his invaluable support in revising the research protocol and his inspiring encouragements from the inception of this study till its completion. Dr Charlotte Hanlon and Dr Abebaw Fekadu in Addis Ababa University, Department of Psychiatry, also deserve special thanks for their vital input, having a worthwhile contributions to shape this paper to be in its present form. We are grateful to Professor Terry B Northcut from Loyola University Chicago, School of Social work (USA), for her kind assistance in editing the language on the final version of the manuscript. We would also like to thank head office of Administration of Refugee and Returnees Affairs (ARRA) in Addis Ababa, and its district office in Shire in Ethiopia for permission to conduct this study. Furthermore, we would like to acknowledge the worthwhile contribution made by healthcare staff of ARRA and Center for Victims of Trauma (CVT) in Shire and Mai Aini refugee camp for their unreserved support during the entire period of data collection, and all the participants of the study who shared their experiences without reservation.

Contributors $B G$ is the principal investigator $(\mathrm{PI})$ of this study. He led in generating the research idea, design and methods of the study, writing the research protocol, validating measures, data collection, analysis, interpretation and writing of the findings. AA has made contribution in revising the research protocol, the research design, validation of measures, analysis and interpretation of data, and critically reviewing the final manuscript. GM made contribution in checking and editing the statistical analysis, critically reviewing the drafted manuscript. All the authors approved the final version.
Funding This study was financially supported by Addis Ababa University and University of Gondar, Ethiopia.

Competing interests None declared.

Patient consent for publication Not required.

Ethics approval This study had ethical clearance from Institutional Ethical Review Board (IRB) of College of Health Sciences, Addis Ababa University. Participants were provided with information sheet about the study regarding its objective, relevance, beneficence, risk, participant's rights and others. Ethical issues as outlined by declaration of Helsinki for human participants in medical research were adhered.

Provenance and peer review Not commissioned; externally peer reviewed.

Data sharing statement All necessary data to understand in manuscript (MS) is included in tables or text within the MS. The row data in SPSS format will be deposited in the Department of Psychiatry, Addis Ababa University (AAU), and can be accessed in accordance with data sharing policy of Institutional Review Board of College of Health Sciences, AAU.

Open access This is an open access article distributed in accordance with the Creative Commons Attribution Non Commercial (CC BY-NC 4.0) license, which permits others to distribute, remix, adapt, build upon this work non-commercially, and license their derivative works on different terms, provided the original work is properly cited, appropriate credit is given, any changes made indicated, and the use is non-commercial. See: http://creativecommons.org/licenses/by-nc/4.0/.

\section{REFERENCES}

1. Idemudia ES, William JK, Boehnke K, et al. Gender differences in trauma and posttraumatic stress symptoms among displaced Zimbabweans in South Africa. J Trauma Stress Disord Treat 2013;2:1340.

2. Brumsey AD, Joseph NT, Myers HF, et al. Modeling the relationship between trauma and psychological distress among HIV-Positive and HIV-Negative Women. Psychol Trauma 2013;5:69-76.

3. Nosheen A, Riaz MN, Batoo N. Cross-cultural study on social support, sense of coherence and outcomes in Pakistan and Germany. Pakistan Journal of Commerce and Social Sciences 2014;8:445-52.

4. Veronese G, Pepe A. Sense of coherence mediates the effect of trauma on the social and emotional functioning of Palestinian health providers. Am J Orthopsychiatry 2014;84:597-606.

5. Sachs E, Rosenfeld B, Lhewa D, et al. Entering exile: trauma, mental health, and coping among Tibetan refugees arriving in Dharamsala, India. J Trauma Stress 2008;21:199-208.

6. Antonovsky A. Unravelling the mystery of health. How people manage stress and stay well. San Francisco: Jossey-Bass, 1987.

7. Pham PN, Vinck P, Kinkodi DK, et al. Sense of coherence and its association with exposure to traumatic events, posttraumatic stress disorder, and depression in eastern Democratic Republic of Congo. $J$ Trauma Stress 2010;23:313-21.

8. Cherewick M, Tol W, Burnham G, et al. A structural equation model of conflict-affected youth coping and resilience. Health Psychol Behav Med 2016;4:155-74.

9. Dalgard OS, Dowrick C, Lehtinen V, et al. ODIN Group. Negative life events, social support and gender difference in depression: a multinational community survey with data from the ODIN study. Soc Psychiatry Psychiatr Epidemiol 2006;41:444-51.

10. Nakash O, Nagar M, Shoshani A, et al. The association between perceived social support and posttraumatic stress symptoms among Eritrean and Sudanese male asylum seekers in Israel. Int J Cult Ment Health 2017;10:261-75.

11. Lazarus RS, Folkman S. Psychological stress and the coping process. New York: Appleton-Century-Crofts, 1984.

12. Voges MA, Romney DM. Risk and resiliency factors in posttraumatic stress disorder. Ann Gen Hosp Psychiatry 2003;2:4.

13. Hooberman J, Rosenfeld B, Rasmussen A, et al. Resilience in trauma-exposed refugees: the moderating effect of coping style on resilience variables. Am J Orthopsychiatry 2010;80:557-63.

14. Almedom AM, Tesfamichael B, Saeed Mohammed Z, et al. Use of 'sense of coherence (SOC)' scale to measure resilience in Eritrea: interrogating both the data and the scale. J Biosoc Sci 2007;39:91-107.

15. Aljurany H. Personality characteristics, trauma and symptoms of PTSD: A population based study in Iraq: Heriot Watt University, 2013. PhD dissertation.

16. Li M. Relationship among sense of coherence, coping strategies, and interpersonal patterns: a cross-cultural study. American Counseling Association 2015;63:1-10. 
17. Miller KE, Rasmussen A. The mental health of civilians displaced by armed conflict: an ecological model of refugee distress. Epidemiol Psychiatr Sci 2017;26:129-38.

18. Araya M, Chotai J, Komproe IH, et al. Gender differences in traumatic life events, coping strategies, perceived social support and sociodemographics among postconflict displaced persons in Ethiopia. Soc Psychiatry Psychiatr Epidemiol 2007;42:307-15.

19. Women's Refugee Commission. Young and astray, an assessment of driving the movement of an accompanied children and adolescents from Eritrea into Ethiopia, Sudan and Beyond. New York: Women's Refugee Commission, 2013.

20. United Nations Higher Commissioner for Refugees. Ethiopia, operational overview: camp demographic population statistics by office and region. https://reliefweb.int/sites/reliefweb.int/files/ resources/Auguststatisticspackage.pdf (As of 31 Aug 2013).

21. United Nations Higher Commissioner for Refugees. Mai Aini refugee camp. Camp profile Shire as of 31st January,2018. UNHCR,Ethiopia. https://data2.unhcr.org/ar/documents/download/ 62694.

22. de Jong JT, Komproe IH, Van Ommeren M, et al. Lifetime events and posttraumatic stress disorder in 4 postconflict settings. JAMA 2001;286:255-62.

23. OnyutLP PL, Neuner F. The Nakivale Camp Mental Health Project: building Local Competency for Psychological assistance to Traumatized Refugees. Intervention 2004;2:90-107.

24. Kamau M, Silove D, Steel Z, et al. Psychiatric disorders in an african refugee camp. Intervention 2004;2:84-9.

25. van Ommeren M, Sharma B, Thapa S, et al. Preparing instruments for transcultural research: use of the translation monitoring form with nepali-speaking bhutanese refugees. Transcult Psychiatry 1999;36:285-301.

26. Lynn MR. Determination and quantification of content validity. Nurs Res 1986;35:382-6.

27. Polit DF, Beck CT. The content validity index: are you sure you know what's being reported? Critique and recommendations. Res Nurs Health 2006;29:489-97.

28. Idemudia ES, Williams JK, Madu SN, et al. Trauma exposures and post traumatic stress among Zimbabwean refugees in South Africa. Life Science Journal 2013;10:2397-497.

29. Radloff LS. The CES-D Scale: a self-report depression scale for research in the general population. Applied Psychological measurement 1977;1:385-401.

30. Moges MF. Translation And Adaptation Of The Center For Epidemiologic Studies-Depression (CES-D) Scale into Tigrigna Language For Tigrigna Speaking Eritrean Immigrants in the United States: University of South Florida, ProQuest Dissertations Publishing, 2011:3464689. Phd Thesis.
31. Prins A, Ouimette P, Kimerling R, et al. The primary care PTSD screen (PC-PTSD): development and operating characteristics. Primary Care Psychiatry 2004;9:9-14.

32. Bliese PD, Wright KM, Adler AB, et al. Validating the primary care posttraumatic stress disorder screen and the posttraumatic stress disorder checklist with soldiers returning from combat. J Consult Clin Psychol 2008;76:272-81.

33. Hoge CW, Auchterlonie JL, Milliken CS. Mental health problems, use of mental health services, and attrition from military service after returning from deployment to Iraq or Afghanistan. JAMA 2006;295:1023-32.

34. Taylor EM, Yanni EA, Pezzi C, et al. Physical and mental health status of Iraqi refugees resettled in the United States. J Immigr Minor Health 2014;16:1130-7.

35. Caitlin M. Somali Refugee Interpretations of trauma-related Mental Illness: Similarities and Differences between the Somali concepts of 'MurugoJoogto' and 'Qulub' and PTSD. Undergraduate Research Journal at the University of Northern Colorado 2013;3:1.

36. Araya M. Post-conflict internally displaced persons in Ethiopia: Mental Distress and Quality of life in relation to Traumatic Life events, Coping Strategy, Social support, and Living Conditions. 2007.

37. Antonovsky A. The structure and properties of the sense of coherence scale. Soc Sci Med 1993;36:725-33.

38. Mahammadzadeh A, Poursharifi H, Alipour A. Validation of Sense of Coherence (SOC) 13-item scale in Iranian sample. Procedia Soc Behav Sci 2010;5:1451-5.

39. Eriksson M, Lindström B. Validity of Antonovsky's sense of coherence scale: a systematic review. J Epidemiol Community Health 2005;59:460-6.

40. Abiola T, Udofia O, Zakari M. Psychometric properties of the 3-Item Oslo Social Support Scale among Clinical Students of Bayero University, Kano, Nigeria. Malaysian Journal of Psychiatry 2013;22:2.

41. Schreiber JB, Nora A, Stage FK, et al. Reporting Structural Equation Modeling and Confirmatory Factor Analysis Results: A Review. $J$ Educ Res 2006;99:323-38.

42. Steel Z, Silove D, Brooks R, et al. Impact of immigration detention and temporary protection on the mental health of refugees. $\mathrm{Br} \mathrm{J}$ Psychiatry 2006;188:58-64.

43. Lincoln KD, Chatters LM, Taylor RJ. Social Support, Traumatic Events, and Depressive Symptoms Among African Americans. J Marriage Fam 2005;67:754-66.

44. Feseha $G, G /$ mariam $A$, Gerbaba M. Intimate partner physical violence among women in Shimelba refugee camp, northern Ethiopia. BMC Public Health 2012;12:1-10.

45. Wirtz AL, Glass N, Pham K, et al. Development of a screening tool to identify female survivors of gender-based violence in a humanitarian setting: qualitative evidence from research among refugees in Ethiopia. Confl Health 2013;7:13. 